\title{
Algas epífitas de Bajo Pepito, Isla Mujeres, Quintana Roo, México
}

\author{
L.I. Quan-Young ${ }^{1}$, M.A. Díaz-Martín \& J. Espinoza-Avalos \\ 1 ECOSUR, Unidad Chetumal, Apdo. Postal 424. Chetumal, Quintana Roo 77000, México. Fax 52(983)8350454, \\ lquan@ecosur-qroo.mx
}

Recibido 15-VI-2000. Corregido 17-V-2001. Aceptado 27-II-2003.

\begin{abstract}
Epiphytic algae from Bajo Pepito, Isla Mujeres, Quintana Roo, México. A total of 96 epiphytic algae species were identified from Bajo Pepito, Quintana Roo, México. 60.4\% (58) belonged to the Rhodophyta, $19.79 \%$ (19) to the Phaeophyta, $16.6 \%$ (16) to the Chlorophyta and $3.1 \%$ (3) to the Cyanophyta; 49 species $(50.5 \%)$ were found only in one month, while Heterosiphonia crispella was found in all of the sampled months. That species provided the largest contribution to the biomass of epiphytes. During January we registered the greater biommass and richness of epiphytes species, coincidently with high values of host species cover and rainfall. Rev. Biol. Trop. 54(2): 317-328. Epub 2006 Jun 01.
\end{abstract}

Key words: epiphytic algae, Caribbean, species richness, biomass.

Para toda la zona del Caribe existen muy pocos trabajos específicos en los que se informan algas epífitas; sin embargo, algunos de los trabajos que podemos mencionar son: Velásquez et al. (1987), quienes informaron 17 macroalgas epífitas para la laguna de Marites en Venezuela; Huerta-Múzquiz et al. (1987) encontraron 133 especies de algas epífitas en un estudio sobre las algas marinas de la Península de Yucatán. En Quintana Roo, Mateo-Cid y Mendoza-González (1991) informaron 114 especies de epífitas para Cozumel, y, específicamente para Isla Mujeres MendozaGonzález y Mateo-Cid (1992) registraron 59 especies de algas epífitas. El objetivo de este trabajo es mejorar el conocimiento sobre algas epífitas del Mar Caribe, ya que en muchos estudios realizados en esta área no se han considerado aparte de aquellas fijas a rocas.

\section{MATERIALES Y MÉTODOS}

El sitio de estudio, Bajo Pepito se encuentra localizado dentro del canal de Yucatán, siendo parte de la zona tropical mexicana del Mar Caribe. Se localiza en la región suroeste de Isla Mujeres, Quintana Roo, México, a una latitud norte de $21^{\circ} 12^{\prime} 21^{\prime \prime}$ y una longitud oeste de $86^{\circ} 44^{\prime} 40^{\prime \prime}$. Posee aguas claras y corrientes marinas fuertes todo el año, con una profundidad entre 3 y $4 \mathrm{~m}$, es una zona rocosa-arenosa, con rocas no mayores de $50 \mathrm{~cm}$ de diámetro.

Las macroalgas epífitas se desprendieron de otras fijas a rocas y que fueron recolectadas en la zona de estudio, de febrero de 1997 a enero de 1998. En el laboratorio las algas epífitas se desprendieron con pinza de relojero y se identificaron con la ayuda de trabajos como los de Taylor (1960), Joly (1967), Earle (1969), Hine (1977), Littler et al. (1989), y Littler y Littler (1997). Para la identificación del material se contó con la ayuda de microscopio estereoscópico y compuesto; algunas veces fue necesario realizar preparaciones permanentes. La actualización nomenclatural de las especies se realizó siguiendo a Wynne (1998). Para determinar la biomasa húmeda de las algas epífitas se eligieron las más conspicuas, se les drenó el exceso de agua y se registró su peso 
en una balanza Sartorius Portable PT/2000 (como algas conspicuas se consideró aquellas que tenían un peso suficiente para registrarse en la balanza utilizada).

\section{RESULTADOS}

Se encontró un total de 96 especies de algas epífitas; 58 Rhodophyta, 19 Phaeophyta, 16 Chlorophyta y 3 Cyanophyta. Del total de algas epífitas que estamos registrando en este trabajo, 49 especies (51\%) se encontraron únicamente en un sólo mes de muestreo, mientras que Heterosiphonia crispella se encontró durante todo el periodo de estudio. Amphiroa fragilissima, Dictyota pinnatifida, Dichotryx sp., Haliptilon cubense y Jania adhaerens también fueron comunes, encontrándose entre el $80 \%$ y $90 \%$ de los meses de muestreo (Cuadro 1).

La mayor riqueza de algas epífitas se encontró en enero (51 especies, Cuadro 1), lo cual podría deberse a un mayor reclutamiento de las especies. La menor riqueza se presentó en los meses de agosto y octubre (14 especies, Cuadro 1). En cuanto a la biomasa, el mayor valor se presentó en enero ( $24 \mathrm{~g}$, Cuadro 2 ) y el menor en abril ( $0.5 \mathrm{~g}$, Cuadro 2). Tanto la mayor riqueza como la mayor biomasa de algas epífitas se presentó en enero, donde ocurren valores altos de riqueza y cobertura de macroalgas hospederas, así como alta precipitación pluvial (Quan-Young et al. 2004). El alga que contribuyó mayormente con la biomasa de epífitas fue Heterosiphonia crispella. Las macroalgas que fueron utilizadas como hospederas por un mayor número de especies epífitas son Digenea simplex (35), Halimeda incrassata (29), Laurencia poiteaui (35), Laurencia obtusa (21) y Udotea flabellum (21), mientras que las algas hospederas que sólo fueron ocupadas por una especie de epífita son: Champia salicornioides, Dasya ramosissima, Heterosiphonia gibbessii, Liagora pinnata, Penicillus pyriformis, Ventricaria ventricosa, Caulerpa webbiana, Udotea cyathiformis y Wrangelia penicillata (Cuadro 3).

\section{DISCUSIÓN}

Se registraron 96 especies de algas epífitas, 58 de ellas pertenecientes a la división Rhodophyta. El número de algas epífitas registrado en este trabajo fue menor al registrado en otros trabajos efectuados en el Caribe mexicano (Huerta-Múzquiz et al. 1980, Mateo-Cid y Mendoza-González 1991). Esto se debe probablemente a que en esos trabajos se incluyeron varias zonas de muestreo, mientras que la información de nuestro estudio corresponde a un solo sitio de colecta. Heterosiphonia crispella fue la única especie que se registró en todos los meses de colecta y fue la que contribuyó mayormente a la biomasa. en enero se presentó la mayor riqueza (51) y la mayor biomasa ( $24 \mathrm{~g}$ ) de macroalgas epífitas, ya que durante la temporada de huracanes y nortes, existe una disminución, tanto en riqueza de especies como en biomasa, debido al fuerte oleaje el cual probablemente desprende del substrato a las algas hospederas. 


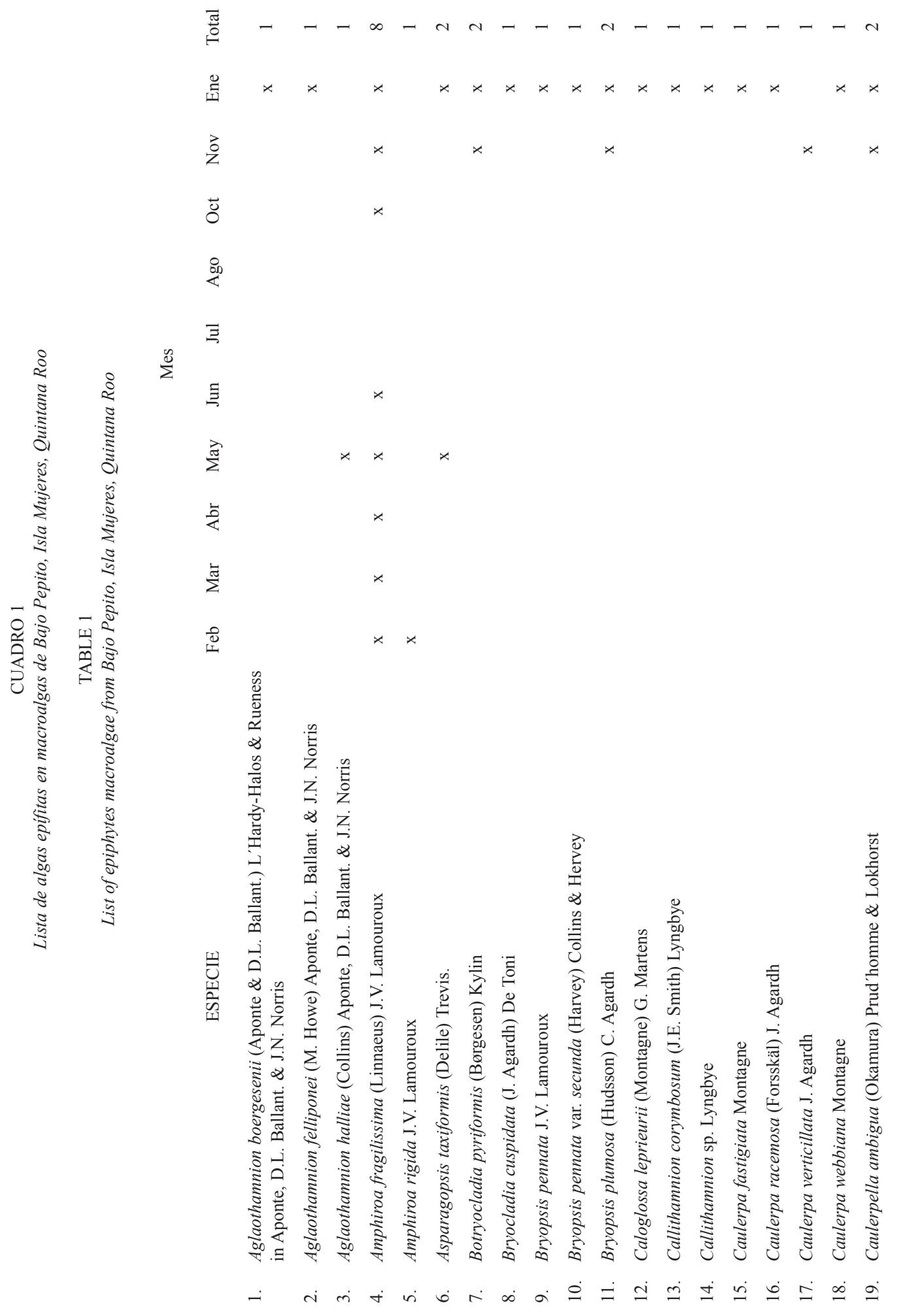




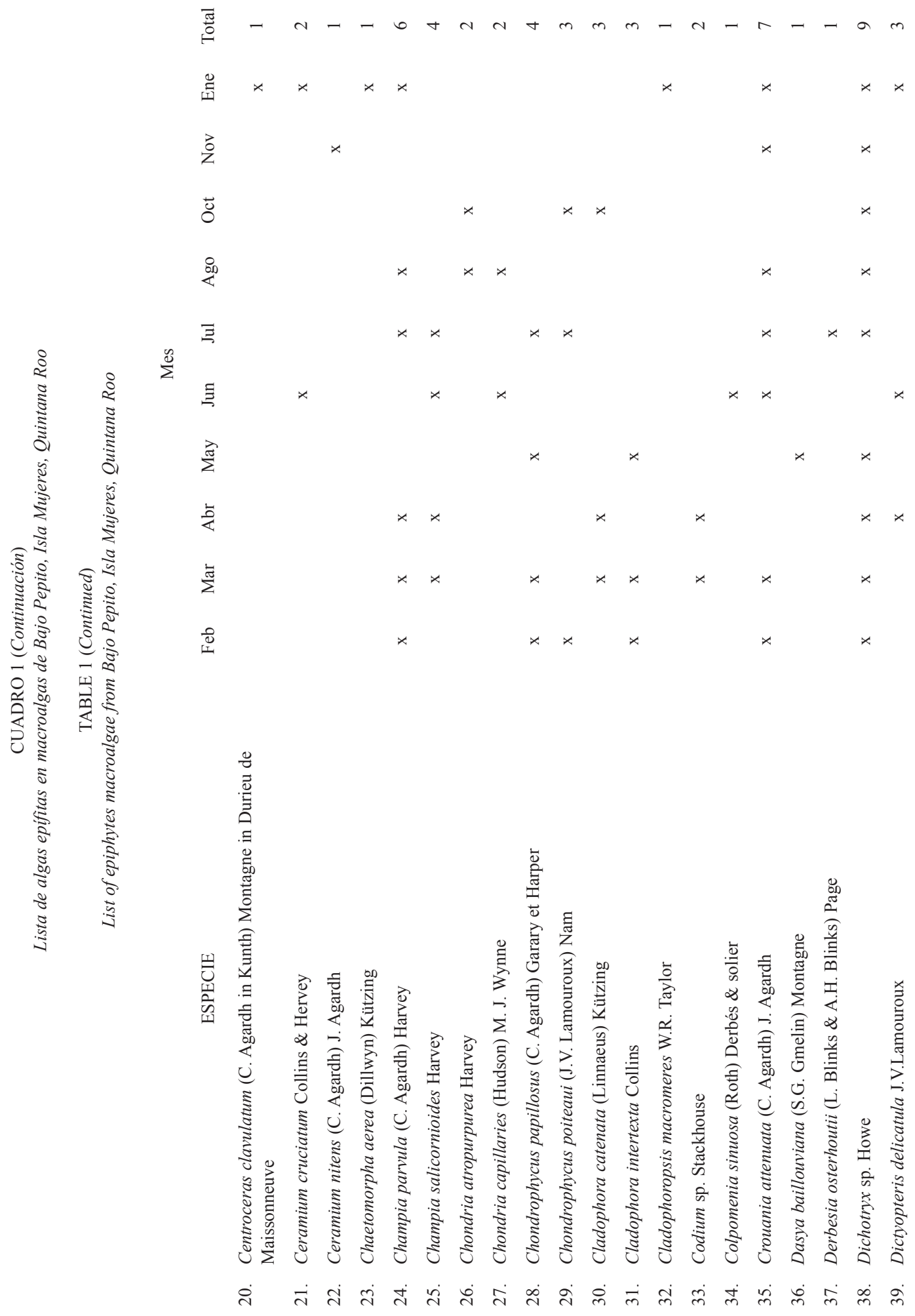




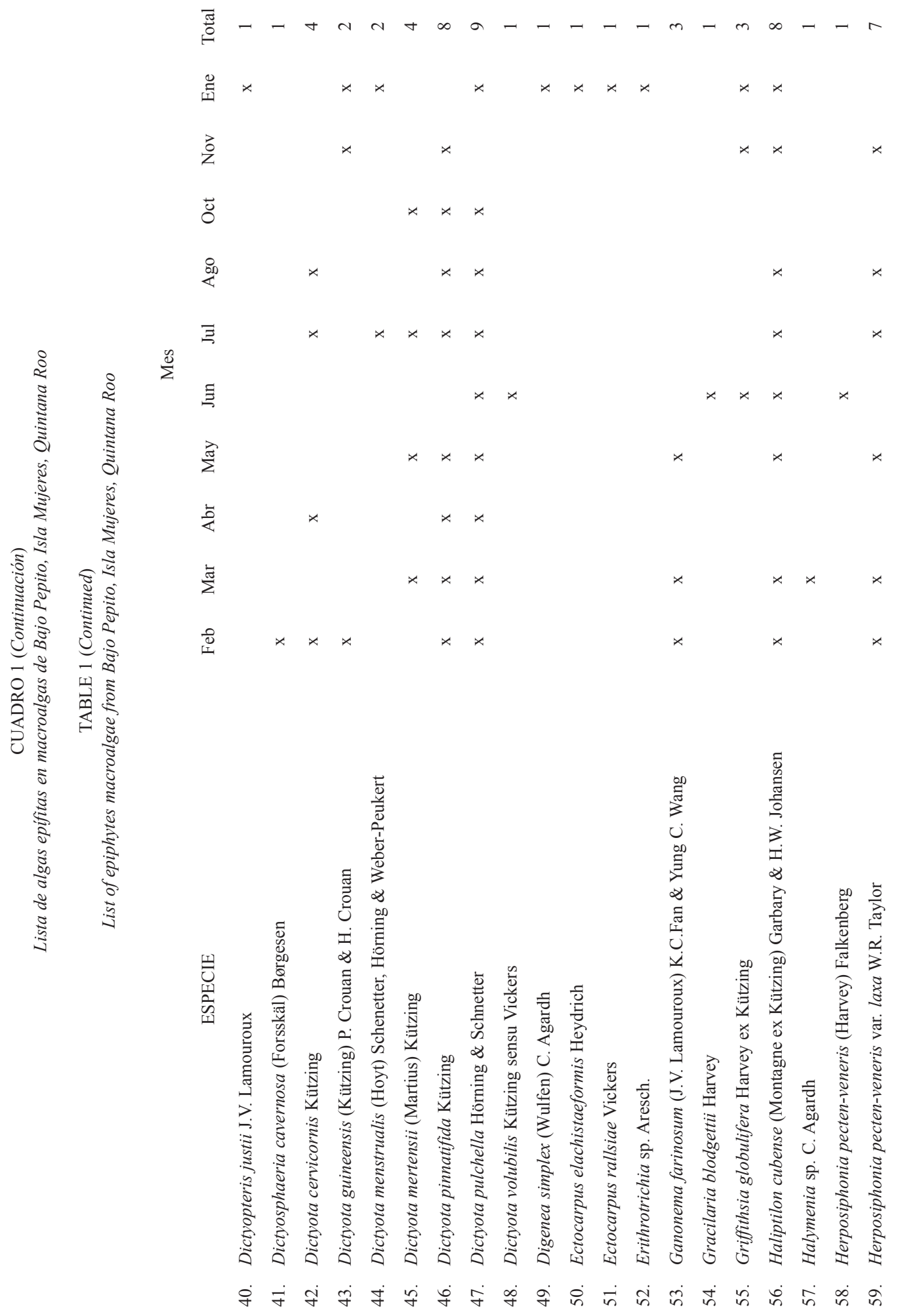




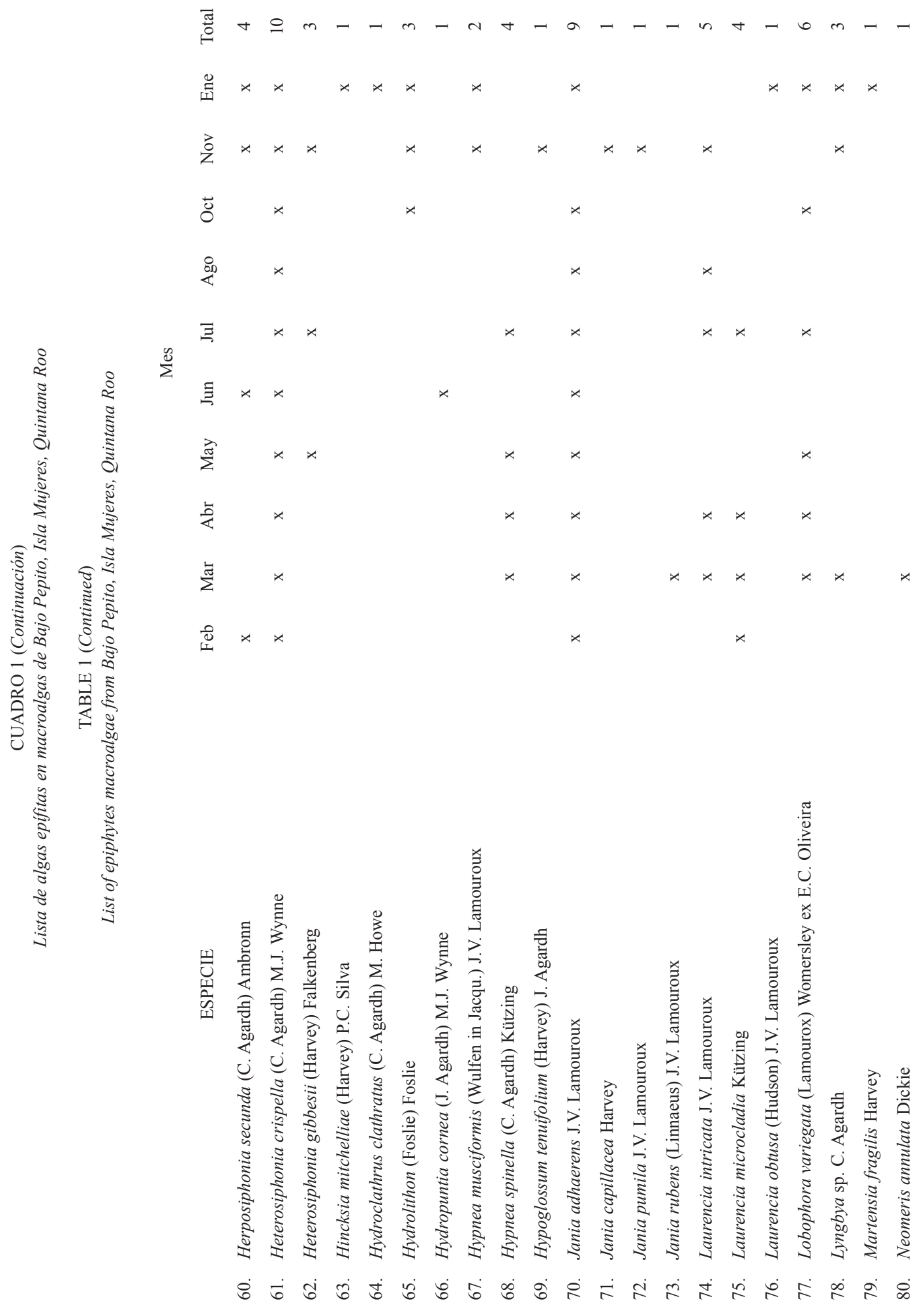




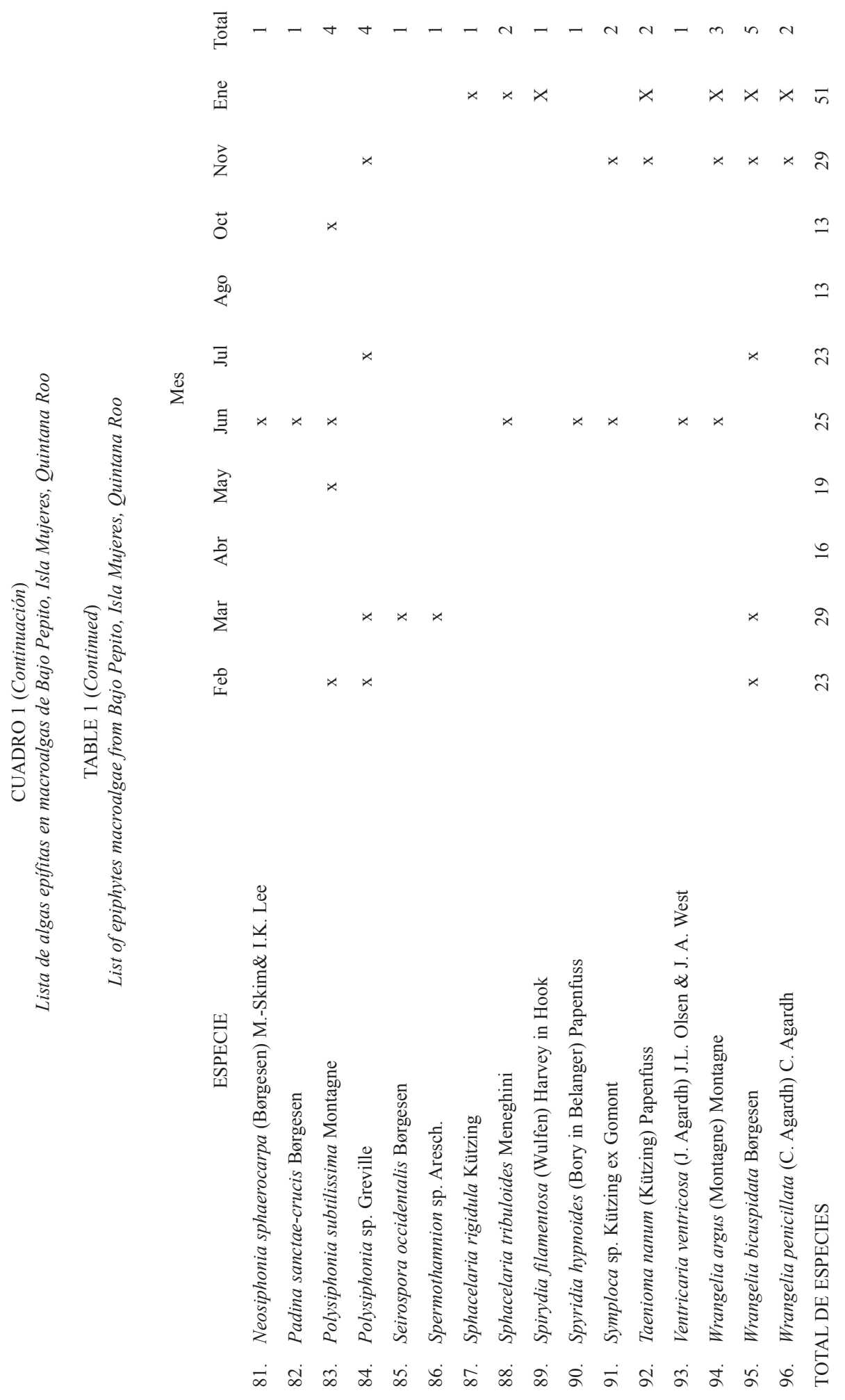




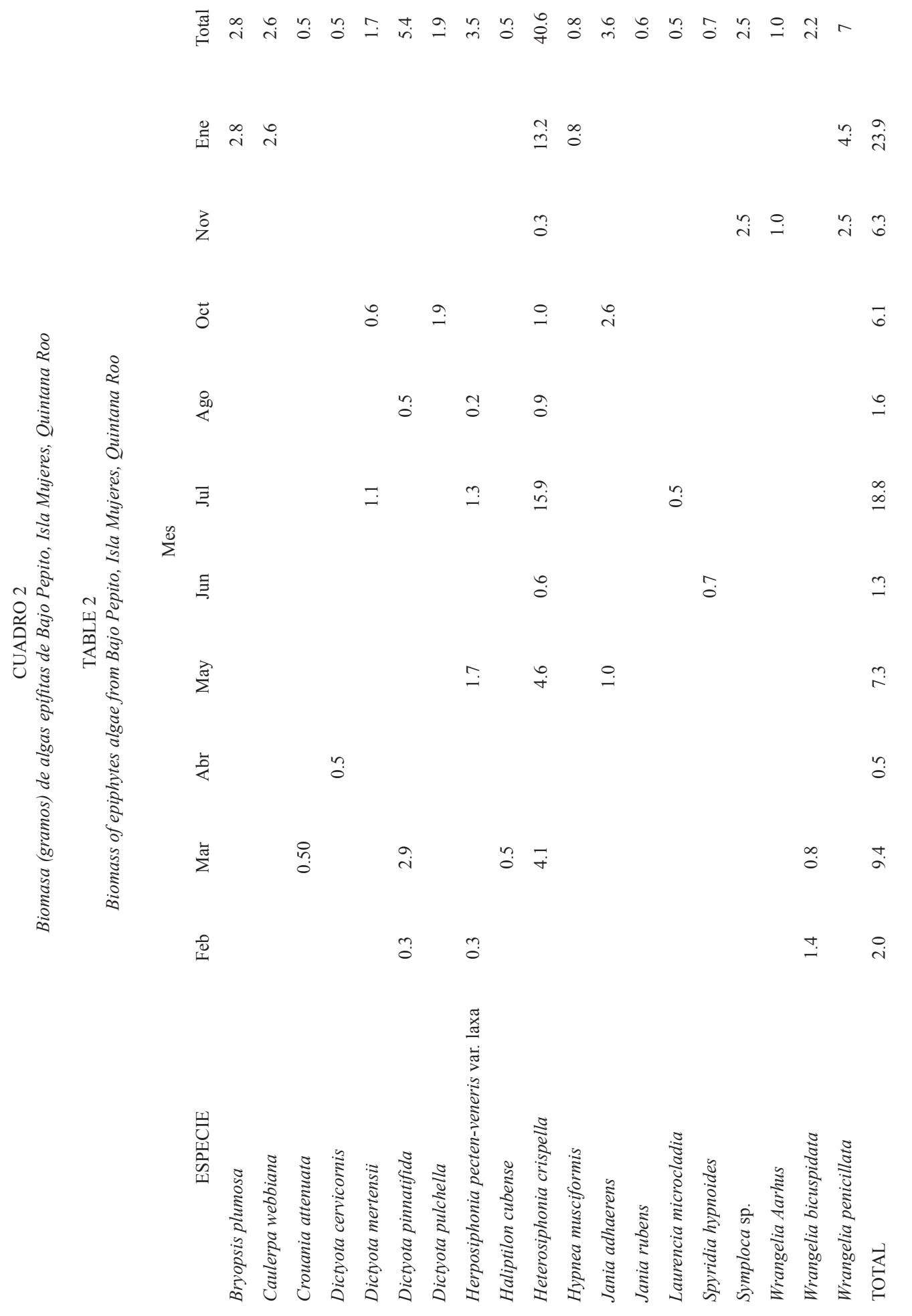



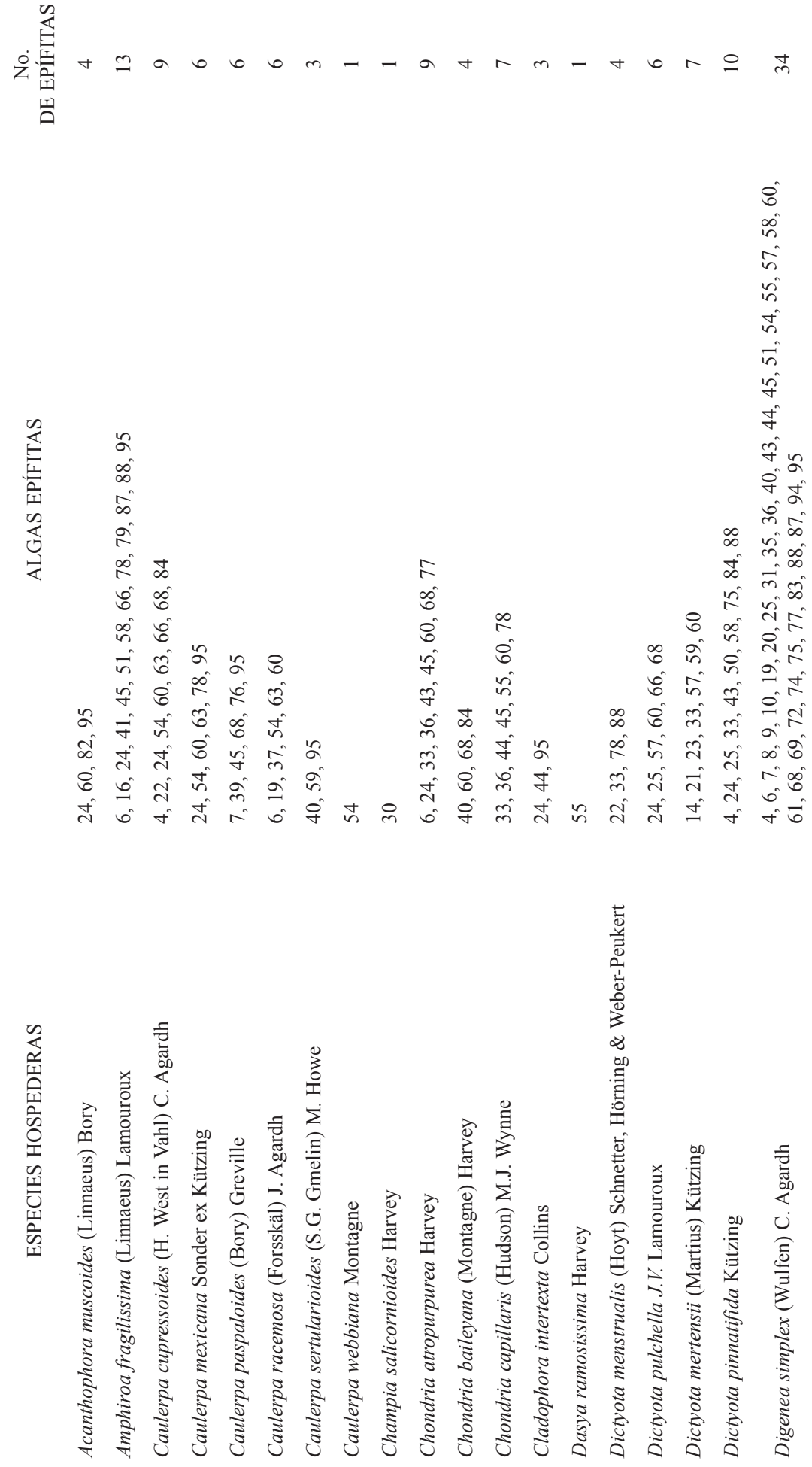

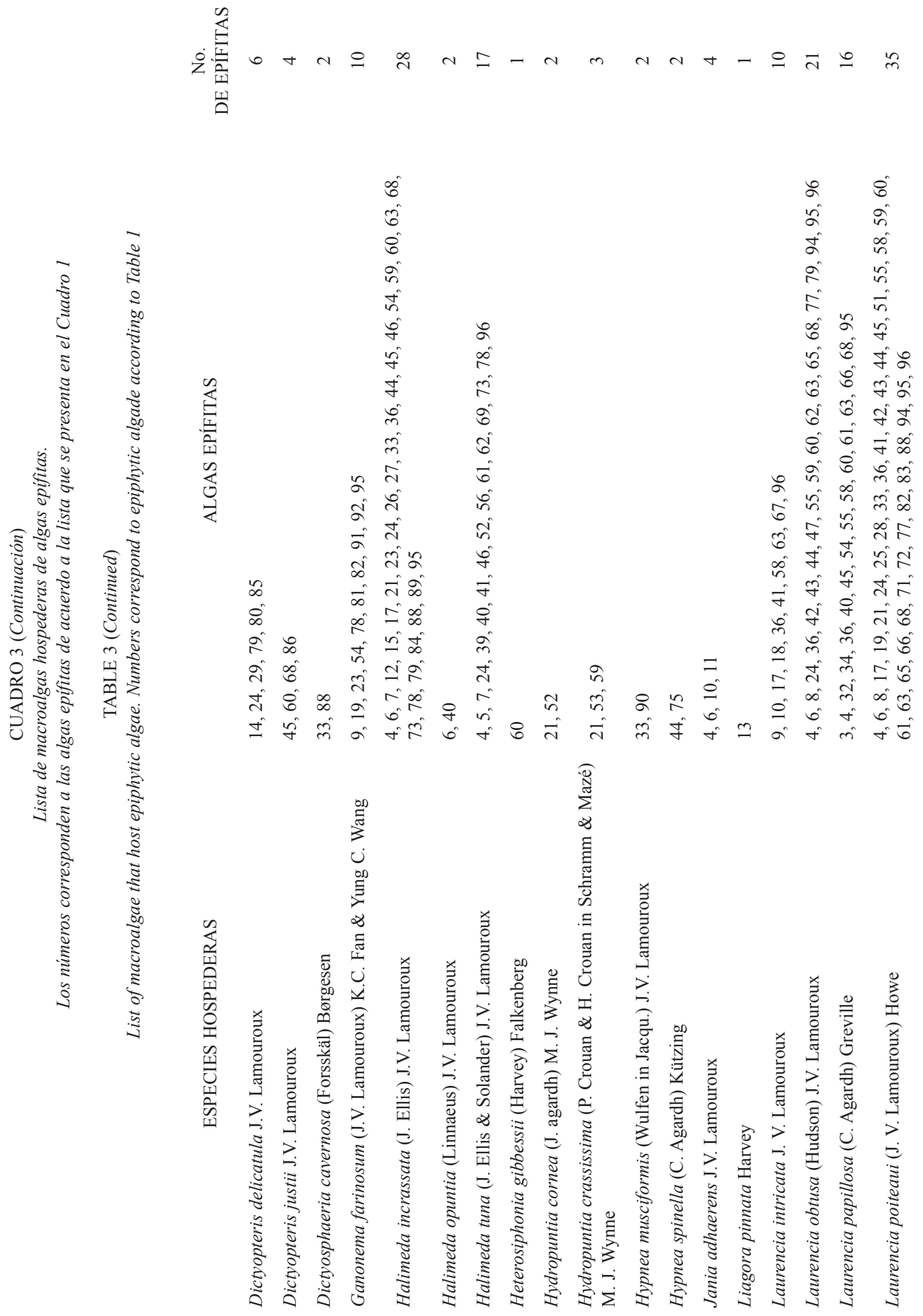


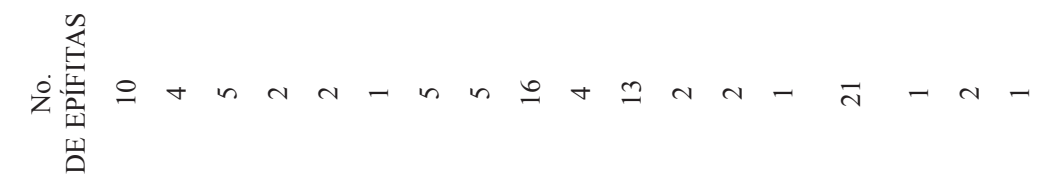

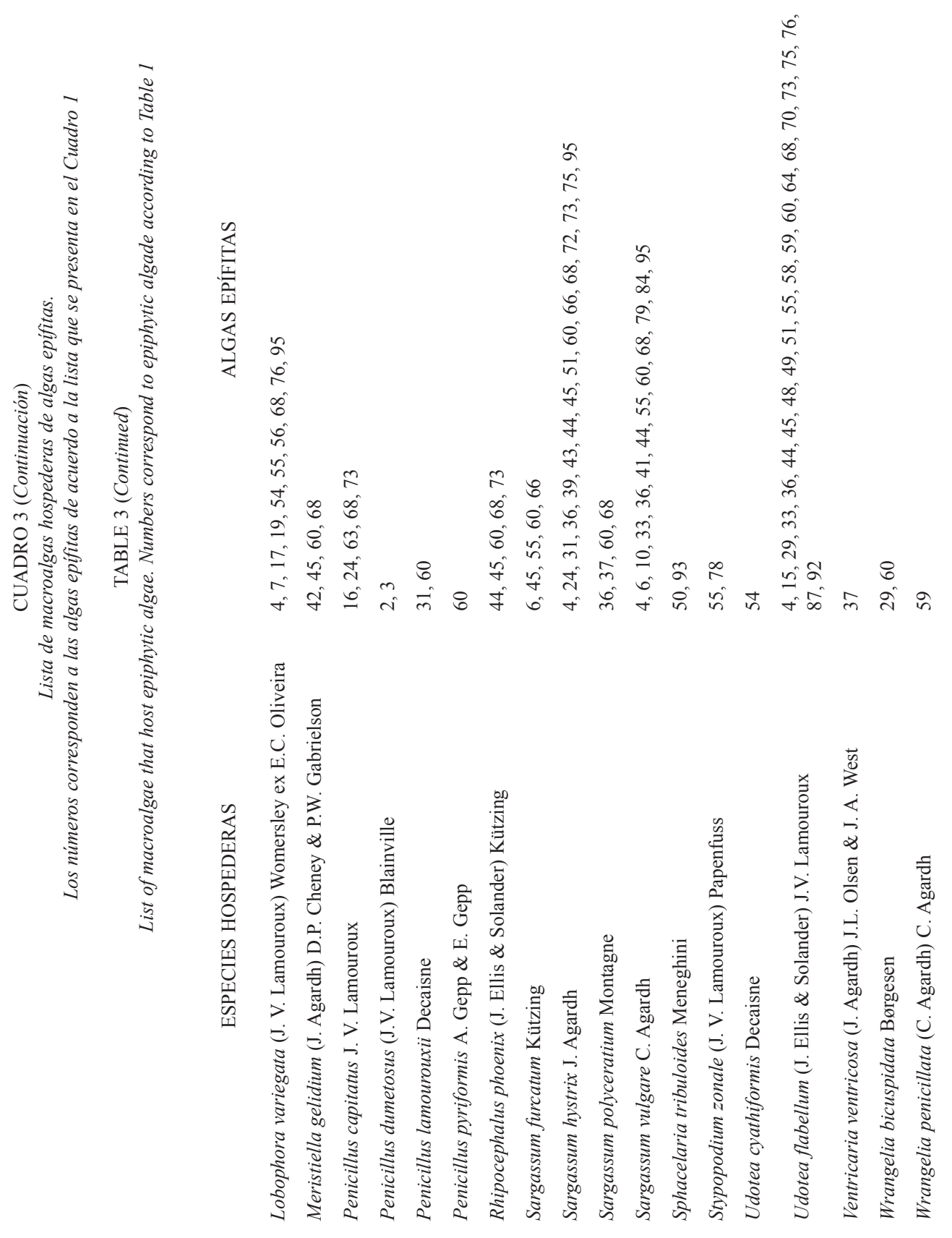




\section{AGRADECIMIENTOS}

Al CONACYT por financiar el trabajo a través del proyecto $0418 \mathrm{P}-\mathrm{T}$ y al ECOSUR.

\section{RESUMEN}

Se identificó un total de 96 especies de algas epífitas de Bajo Pepito, Quintana Roo, México; el $60.4 \%$ (58) pertenecieron a la división Rhodophyta, $19.79 \%$ (19) a la división Phaeophyta, $16.6 \%$ (16) a la división Chlorophyta y $3.1 \%$ (3) a la división Cyanophyta; 49 especies $(51 \%)$ se presentaron solamente en un mes de muestreo. Heterosiphonia crispella se presentó en todos los meses de muestreo, y fue la que tuvo mayor contribución en la biomasa de epífitas. En enero se registró la mayor biomasa y riqueza de algas epífitas, lo cual coincidió con valores altos de cobertura de especies hospederas y precipitación pluvial.

Palabras clave: algas epífitas, Caribe, riqueza de especies, biomasa.

\section{REFERENCIAS}

Earle, S.A. 1969. Phaeophyta of the eastern Gulf of Mexico. Phycology 7(2): 71-254.

Hine, A.E. 1977. A glossary of phycological terms for student of marine macroalgae. Alden's in the weeds. Miami, Florida, EEUU. 90 p.

Huerta-Múzquiz, L., A.C. Mendoza-González \& L.E. Mateo-Cid. 1987. Avance sobre un estudio de algas marinas de la Península de Yucatán. Phytology 62: $22-53$.

Joly, A.B. 1967. Generos de algas marinhas da costa atlantica latinoamericana. Universidade de Sao Paulo, Sao Paulo, Brasil. 461 p.

Littler, D.S. \& M.M. Littler. 1997. An illustrated marine flora of the Pelican Cays, Belize. Bull. Biol. Soc. Wash. 9:1-149.

Littler, D.S., M.M. Littler, K.M. Bucher \& J.N. Norris. 1989. Marine plants of the Caribbean: a field guide from Florida to Brasil. Smithsonian Institution, Washington, D.C., EEUU. 263 p.

Mateo-Cid, L.E. \& A.C. Mendoza-González. 1991. Algas marinas bénticas de la isla Cozumel, Quintana Roo, México. Acta Bot. Mex. 16: 57-87.

Mendoza-González, A.C. \& L.E. Mateo-Cid. 1992. Algas marinas bentónicas de Isla Mujeres, Quintana Roo, México. Acta Bot. Mex 19: 37-61.

Quan Young, L.I., M.A. Díaz-Martín \& J. EspinozaAvalos. 2004. Floristic, cover and phenology of marine macroalgae from Bajo Pepito, Isla Mujeres, Mexican Caribbean. Bull. Mar. Sci. 75: 11-25

Taylor, W.R. 1960. Marine algae of the eastern tropical and subtropical coasts of the americas. The University of Michigan, Michigan, EEUU. 1289 p.

Velásquez, Y., L. Valle \& N. Otaola. 1987. Macro-algas bentónicas y epífitas en la laguna Marites, Isla Margarita, Venezuela. Memoria de la Sociedad de Ciencias Naturales La Salle. 48: 25-37.

Wynne, M.J. 1998. A checklist of benthic marine algae of the tropical and subtropical western Atlantic. Nova Hedwigia, Beiheft 116: 1-155. 